\title{
Numerical modelling of frequency comb generation in nonlinear resonators
}

\author{
M. Erkintalo*, F. Leo*, T. Hansson ${ }^{\dagger,}$, K. E. Webb*, S. G. Murdoch*, J. Anthony*, \\ I. Ricciardi ${ }^{\S}$, M. De Rosa ${ }^{\S}$, S. Wabnitz ${ }^{\ddagger}$, , and S. Coen* \\ *The Dodd-Walls Centre, Department of Physics, The University of Auckland, Auckland 1142, New Zealand \\ ${ }^{\dagger}$ Department of Applied Physics, Chalmers University of Technology, SE-41296 Göteborg, Sweden \\ ${ }^{\ddagger}$ Dipartimento di Ingegneria dell’Informazione, Università di Brescia, via Branze 38, 25123 Brescia, Italy

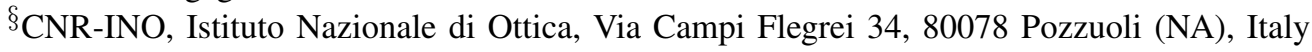 \\ Email: m.erkintalo@ auckland.ac.nz
}

\begin{abstract}
We present a comprehensive review of recent work on numerical modelling of frequency comb generation in coherently-driven nonlinear resonators. Specifically, we discuss modelling and nonlinear dynamics of frequency combs generated in Kerr nonlinear microresonators, emphasizing links to similar dynamics studied in macroscopic fibre cavities. In addition to established Kerr frequency comb generators, we will also discuss emerging comb generation platforms based on continuouslydriven quadratically nonlinear resonators.
\end{abstract}

\section{INTRODUCTION}

Optical frequency combs are light sources whose spectrum is composed of numerous equidistantly-spaced narrow components [1]. They have a wide range of applications across the natural sciences, including precision spectroscopy, calibration of astronomical spectrographs, and all-optical atomic clocks. Commercially available comb generators are based on modelocked lasers and fibre supercontinuum generation: the spectral comb structure ensues from the periodicity of the mode-locked pulse train. However, in 2007 Del'Haye et al. demonstrated a new approach to create frequency combs with extraordinary characteristics complementary to those achievable using mode-locked lasers [2]. Specifically, the researchers showed that a low-power monochromatic continuous wave (cw) laser coupled into a high-Q microresonator could transform into a frequency comb whose line-spacing was governed by the resonator free-spectral range (FSR). The ability to use lowpower cw lasers and microscopic structures to create frequency combs with hundreds of $\mathrm{GHz}$ line-spacing quickly attracted widespread interest [3].

Microresonator frequency combs physically arise via thirdorder "Kerr" nonlinear effects, namely degenerate and nondegenerate four-wave mixing [4], [5]. Although not fully appreciated until recently [6], the underlying physics is in fact closely related to other realizations of coherently-driven Kerrnonlinear cavities, including spatially diffractive cavities [7] and macroscopic fibre-optic ring resonators [8]-[10]. Notably, the different systems obey similar general master equations and display similar nonlinear dynamics [11], [12]. This comparatively recent observation has been pivotal in allowing for the elucidation of the dynamics that underpin the generation of frequency comb in Kerr microresonators.

Although all microresonator frequency comb studies to date have revolved around third-order Kerr nonlinear effects, recent studies in macroscopic, free-space cavities have shown that similar phenomenologies can also manifest themselves in quadratically nonlinear cavities [13], [14]. In particular, experiments have shown that, similarly as for Kerr cavities, a cw laser coupled into a quadratically nonlinear cavity can transform into a frequency comb [14]. The ability to leverage second-order effects for frequency comb generation holds great promise to enable sources at spectral regions that are difficult to access with alternative comb technologies, such as the visible and mid-infrared spectral ranges [15], [16].

Here, we will review the numerical modelling of resonatorbased frequency comb sources. Specifically, we will discuss in detail the master equations governing Kerr microresonator frequency combs, and also elaborate on the underlying nonlinear dynamics. We will also describe the modelling and dynamics of emerging quadratically nonlinear comb sources.

\section{RESULTS}

The evolution of the slowly-varying electric field envelope $E(t, \tau)$ inside a high-finesse, cw-laser-driven Kerr nonlinear resonator is governed by the driven-damped nonlinear Schrödinger equation [6]:

$$
\begin{aligned}
t_{\mathrm{R}} \frac{\partial E(t, \tau)}{\partial t} & =\left[-\alpha-i \delta_{0}+i L \sum_{k \geq 2} \frac{\beta_{k}}{k !}\left(i \frac{\partial}{\partial \tau}\right)^{k}\right] E \\
& +i \gamma L|E|^{2}+\sqrt{\theta} E_{\mathrm{in}}
\end{aligned}
$$

Here $t_{\mathrm{R}}$ is the roundtrip time, $\alpha=\left(\alpha_{\mathrm{i}} L+\theta\right) / 2$ describes the total cavity losses, $\delta_{0}=2 \pi l-\phi_{0}$ is the phase detuning of the driving field with respect to the closest linear resonance (with order $l$ ), $L$ is the cavity length, $\beta_{k}$ are the linear dispersion coefficients, $\gamma$ is the nonlinearity coefficient and $\theta_{1}$ is the power transmission coefficient of the coupler used to inject the driving field $E_{\text {in }}$ into the cavity. The continuous variable $t$ is a slow time that measures the evolution of the field envelope over several roundtrips, whilst $\tau$ is a fast time variable that allows for the description of the field profile. When neglecting 
(a)

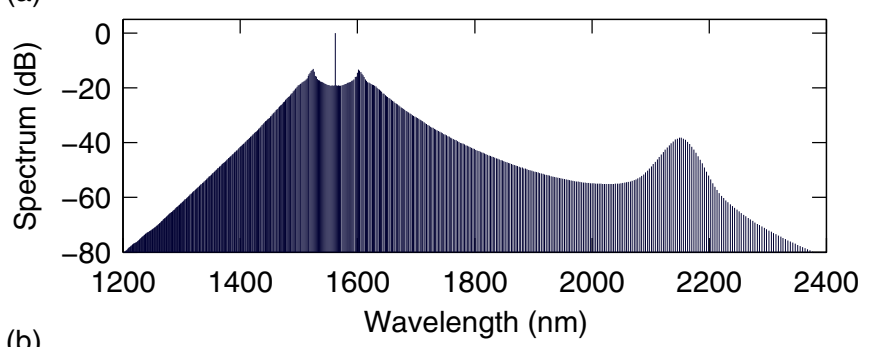

(b)

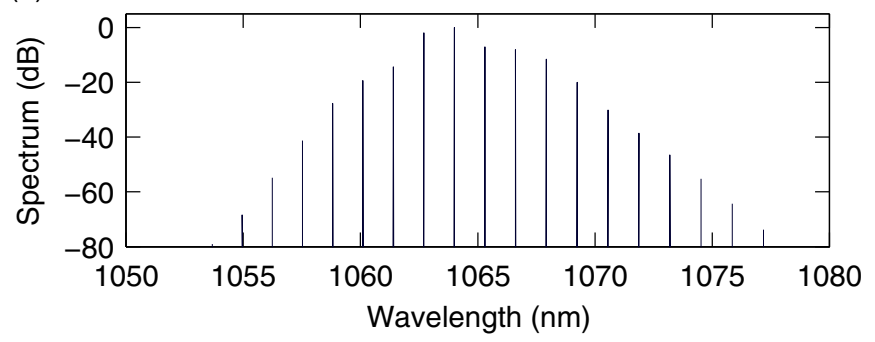

Fig. 1. (a) Numerically simulated frequency comb in a Kerr nonlinear silicon nitride resonator. (b) Numerically simulated frequency comb in a singlyresonant, macroscopic SHG system. For details and parameters, see [18] and [15], respectively.

higher-order dispersion terms $\left(\beta_{k}=0\right.$ for $\left.k>2\right)$, Eq. (1) is formally identical with the Lugiato-Lefever equation used in the study of pattern formation in spatially diffractive Kerr cavities [7]. The same equation also describes coherentlydriven macroscopic fibre cavities, and it has been widely used in that context [8]-[10].

If the resonator also hosts materials that exhibit a secondorder quadratic nonlinearity, the evolution of the field envelope can be modelled using an infinite-dimensional map consisting of a generalized envelope equation and appropriate boundary conditions [17]. However, if a particular second-order process dominates the dynamics, the problem can be greatly simplified by introducing a set of distinct field envelopes with widelyspaced carrier frequencies. For the particular case of secondharmonic generation (SHG) in high-finesse cavities, where frequency combs have been experimentally observed [14], the second-harmonic field is found to be dynamically slaved to the fundamental field, with the latter evolving according to the following mean-field equation [15], [16]:

$$
\begin{aligned}
t_{\mathrm{R}} \frac{\partial E(t, \tau)}{\partial t}= & {\left[-\alpha-i \delta+i L \sum_{k \geq 2} \frac{\beta_{k}}{k !}\right] E } \\
& -(\kappa L)^{2} E^{*}\left[E^{2}(t, \tau) \otimes I(\tau)\right]+\sqrt{\theta} E_{\mathrm{in}} .
\end{aligned}
$$

In addition to the parameters already introduced in (1), $\kappa$ describes the strength of the quadratic nonlinearity, $\otimes$ denotes convolution and $I(\tau)$ is a nonlinear response function that incorporates the resonant cavity configuration as well as the interaction with the second-harmonic field. Notably, the functional form of $I(\tau)$ is different for singly- and doubly-resonant cavity constructions (see [15] and [16] for details).

Equations (1) and (2) can be numerically integrated using a split-step Fourier -type method so as to model the dynamics of frequency comb formation in corresponding resonator configurations. Figure 1(a) and (b) show examples of modelled frequency combs in a Kerr microresonator and in a cavity-enhanced SHG system, respectively. (For simulation parameters, see refs. [18] and [15].) The numerical modelling results are in very good agreement with the corresponding experimentally observed combs reported in refs. [19] and [14], respectively.

\section{CONCLUSION}

We have presented model equations that allow for the theoretical study of frequency comb generation in different cavity configurations. Analysis of the equations allows for straightforward exploration of new designs and settings for improved frequency comb sources. Moreover, examination of the steady-state solutions of the appropriate evolution equations can provide indispensable general insights into the underlying nonlinear dynamics.

\section{REFERENCES}

[1] T. Udem, R. Holzwarth, and T. W. Hänsch, Nature 416, 233 (2002).

[2] P. Del'Haye, A. Schliesser, O. Arcizet, T. Wilken, R. Holzwarth, and T. J. Kippenberg, Nature 450, 1214 (2007).

[3] T. J. Kippenberg, R. Holzwarth, and S. A. Diddams, Science 332, 555 (2011).

[4] Y. K. Chembo, D. V. Strekalov, and N. Yu, Phys. Rev. Lett. 104, 103902 (2010).

[5] T. Hansson, D. Modotto, and S. Wabnitz, Phys. Rev. A 88, 023819 (2013).

[6] S. Coen, H. G. Randle, T. Sylvestre, and M. Erkintalo, Opt. Lett. 38, 37 (2013).

[7] L. A. Lugiato and R. Lefever, Phys. Rev. Lett. 58, 2209 (1987).

[8] M. Haelterman, S. Trillo, and S. Wabnitz, Opt. Commun. 91, 401 (1992).

[9] F. Leo, S. Coen, P. Kockaert, S.-P. Gorza, P. Emplit, and M. Haelterman, Nature Photon. 4, 471 (2010).

[10] J. K. Jang, M. Erkintalo, S. G Murdoch, and S. Coen, Opt. Lett. 39 5503 (2014).

[11] F. Leo, L. Gelens, Ph. Emplit, M. Haelterman, and S. Coen, Opt. Express 219180 (2013).

[12] S. Coen and M. Erkintalo, Opt. Lett. 38, 1790 (2013).

[13] V. Ulvila, C. R. Phillips, L. Halonen, and M. Vainio, Opt. Lett. 38, 4281 (2013).

[14] I. Ricciardi, S. Mosca, M. Parisi, P. Maddaloni, L. Santamaria, P. De Natale, and M. De Rosa, Phys. Rev. A 91, 063839 (2015).

[15] F. Leo, T. Hansson, I. Ricciardi, M. De Rosa, S. Coen, S. Wabnitz, and M. Erkintalo, Phys. Rev. Lett. 116, 033901 (2016).

[16] F. Leo, T. Hansson, I. Ricciardi, M. De Rosa, S. Coen, S. Wabnitz, and M. Erkintalo, arXiv:1602.03517 (2016).

[17] T. Hansson, F. Leo, M. Erkintalo, J. Anthony, S. Coen, I. Ricciardi, M. De Rosa, S. Wabnitz, arXiv:1602.08087 (2016).

[18] M. Erkintalo and S. Coen, Opt. Lett. 39283 (2014).

[19] Y. Okawachi, K. Saha, J. S. Levy, Y. H. Wen, M. Lipson, A. L. Gaeta, Opt. Lett. 36, 3398 (2011). 